\title{
Long-term follow-up of a severely traumatized leg treated with ipsilateral fracture-united fibular transfer in a patient with amputation of the contralateral leg: a case report
}

\author{
Eon Su Kim, Chae Eun Yang, Jiye Kim, Sug Won Kim \\ Department of Plastic and Reconstructive Surgery, Yonsei University Wonju College of Medicine, Wonju Severance Christian Hospital, Wonju, \\ Korea
}

\begin{abstract}
Extensive bone loss associated with severe vascular injury remains a challenge for lower extremity reconstruction. The fibular free flap has been utilized for many decades to reconstruct long-segment tibial defects. We present an unusual scenario of unilateral weightbearing, wherein we salvaged the sole lower extremity by transfer of the fractured ipsilateral fibula and a bipedicled skin flap. A 38-year-old man sustained a severe crush injury in the right leg with loss of circulation. His left lower leg had a soft tissue defect measuring $20 \times 15$ $\mathrm{cm}$ with an exposed comminuted fracture and a $17-\mathrm{cm}$ tibial defect, along with a segmental fracture of the fibula. Subsequently, we reconstructed the tibial defect by transferring a 17-cm-long section of the ipsilateral fibula. We covered the soft tissue defect with a bipedicled skin flap. The patient eventually began to ambulate independently after surgery.
\end{abstract}

Keywords Fibula / Lower extremity / Pedicled flaps / Tibial defect

\author{
Correspondence: Sug Won Kim \\ Department of Plastic and \\ Reconstructive Surgery, Yonsei \\ University Wonju College of Medicine, \\ Wonju Severance Christian Hospital, \\ 20 Ilsan-ro, Wonju 26426, Korea \\ Tel: $+82-33-741-1371$ \\ Fax: +82-33-742-4022 \\ E-mail: sugwonkim@yonsei.ac.kr
}

Received: May 30, 2021 • Revised: August 13, 2021 - Accepted: September 14, 2021

pISSN: 2234-6163 • elSSN: 2234-6171 • https://doi.org/10.5999/aps.2021.01186• Arch Plast Surg 2021;48:699-702

\section{INTRODUCTION}

Various techniques have been described for tibial reconstruction; however, reconstruction of post-traumatic composite tibial and soft tissue defects remains challenging. A fibular free flap is currently considered the standard treatment for tibial defects $>6 \mathrm{~cm}$ [1]. However, in cases where the contralateral fibula cannot be used, an ipsilateral fibular transfer is the alternative, although it can lead to further limb instability [1-4]. We report an unusual case, wherein a composite tibial defect was reconstructed with ipsilateral fractured fibular segments and a bipedicled flap, in a patient who had lost the contralateral leg due to above-knee amputation performed for a severe crush injury.

\section{CASE}

A 38-year-old man sustained injuries in both lower extremities in a motor vehicle accident. Bilateral closed femoral shaft fractures were present, along with bilateral tibio-fibular fractures and severe soft tissue injury. The right leg was amputated at the above-knee level on an emergent basis, due to extensive comminution and loss of circulation caused by the crush injury. The bilateral femoral shaft fractures were fixed with plates. Reduction and external fixation of the left tibia was performed along with titanium plating. Initially, the size of the soft tissue defect was $20 \times 15 \mathrm{~cm}$, and the fractured tibial segments were exposed. The patient was treated in the emergency intensive care unit for 
2 weeks due to multiple rib fractures and hemothorax. A bacterial culture from the tibial defect site revealed the presence of Pseudomonas aeruginosa. Wound care was performed with betadine, saline solution irrigation, and betadine-soaked dressings. The patient was shifted to the general ward 2 weeks after the trauma, at which time debridement and ostectomy of the necrotic tibia were performed. The tibial defect measured $17 \mathrm{~cm}$. A vascularized bone graft was required to reconstruct the tibia; however, the contralateral fibula was lost due to the trauma and the ipsilateral fibula had multiple segmental fractures. We conserved the defect by external fixation of the tibia until complete union of the fractured fibula was achieved.

The $P$. aeruginosa infection did not resolve despite intravenous antibiotic injections and debridement. The patient was transferred to the isolation ward due to neutropenic fever, 7 weeks after the trauma. Carbapenem and other analgesics that were as-

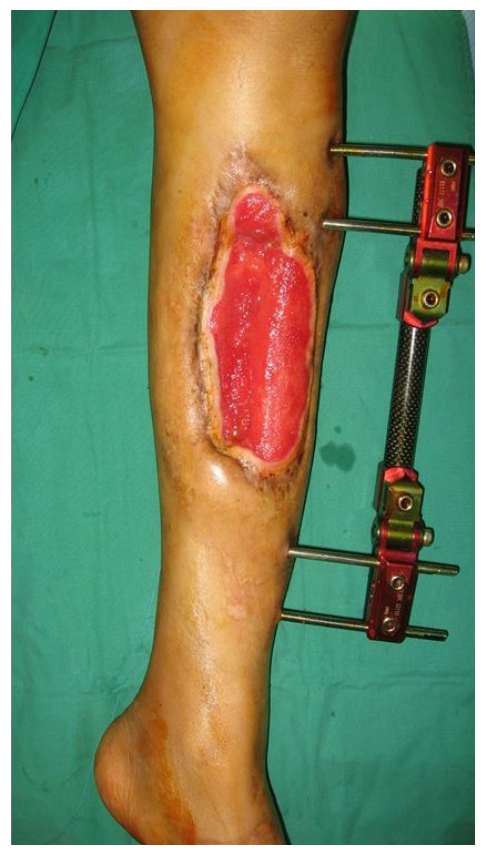

Fig. 1. Four months post-trauma. A $20 \times 15 \mathrm{~cm}$ soft tissue defect can be seen. sumed to have caused the neutropenic fever were discontinued. After 1 week of observation, the patient returned to the general ward. Thorough sterile irrigation and dressing, as well as debridement, were performed under general anesthesia twice in order to eradicate $P$. aeruginosa without antibiotics. During the conservative management period, union of the fractured fibula was observed on serial follow-up radiographs.

Four months after the trauma, decrease of $P$. aeruginosa was observed in wound culture from a $20 \times 15 \mathrm{~cm}$ skin defect, and a united fibula was noted on radiography (Fig. 1). We reconstructed the tibial defect using the ipsilateral fibula, including the united fracture segments.

First, computed tomography angiography was performed before surgery to check whether the three main arteries of the lower extremities were intact. The fibula was approached through a curvilinear posterior incision. The lateral head of the gastrocnemius was mobilized from the distal femur and the soleus was stripped from its origin from the posterior aspect of the fibula and the interosseus membrane. The popliteal vessels were dissected distally to the tibial vessels and the peroneal artery, which supplied the pedicle to the fibula. The fibula was completely exposed and ostectomy was done containing the flexor hallucis longus muscle and the peroneal artery. The $17-\mathrm{cm}$ vascularized fibular flap was transposed through the intermuscular septum. After the transposition of the vascularized fibular flap, a bipedicled flap was transposed from the medial to the anterior side to cover the area of bone exposure. The harvested fibula was affixed to the tibial defect site using a plate and the external fixator system was maintained for stable alignment of the tibia (Fig. 2). The size of the soft tissue defect was $20 \times 15 \mathrm{~cm}$, and it was covered with the bipedicled skin flap.

Two months after surgery, the flap was stable and bony callus formation was observed on radiography. The patient started non-weight-bearing and muscle-strengthening exercises of the left lower leg. Three months postoperatively, hypertrophy of the transferred fibula and complete healing of the femur was ob-
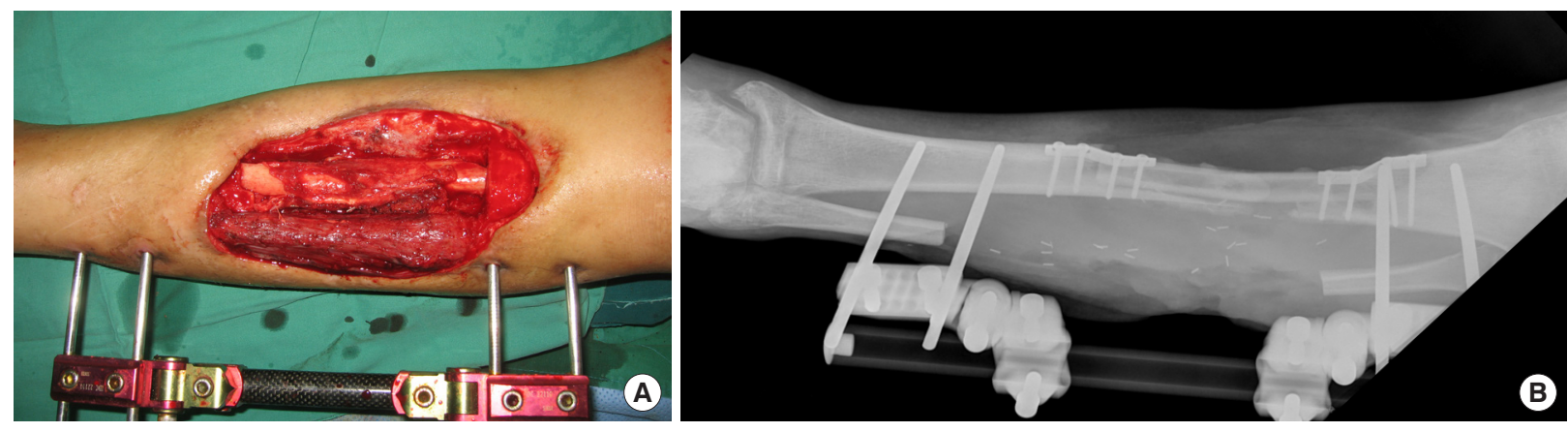

Fig. 2. The fractured fibula was transferred medially to bridge the tibial defect. (A) Clinical photography. (B) Immediate postoperative X-ray. 

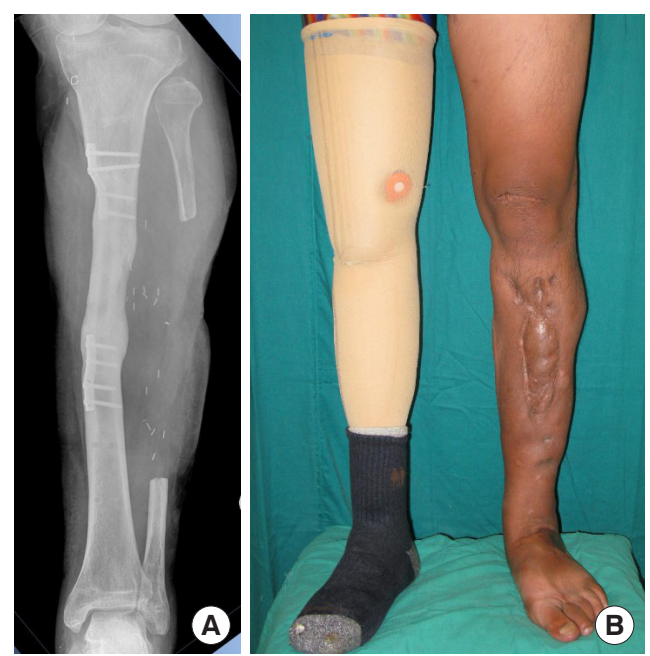

Fig. 3. Two years postoperatively, good bony union and fibular hypertrophy were achieved. (A) X-ray photography. (B) Anteroposterior photograph of the left lower limb showing weight-bearing ability.

served on radiography. Weight-bearing training was started with a lower limb prosthesis on the amputated side. The patient learned to take steps with the prosthesis and the reconstructed lower leg with the help of a walker. Two weeks after the start of weight-bearing exercises, the patient had a fracture in the tibia at the weak point of the proximal plate fixation site. After confirming fracture union, weight-bearing training was restarted with a walker. Subsequently, the patient began to ambulate independently without the walker and returned to routine activities 6 weeks after the second tibial fracture. Two years postoperatively, good bony union and fibular hypertrophy were observed (Fig. 3). We confirmed the patient's satisfaction in performing daily living activities with the reconstructed leg at a 12-year follow-up (Fig. 4).

\section{DISCUSSION}

There are various methods to reconstruct bone defects, such as direct bone grafting, bone transport using the Ilizarov lengthening technique, and using vascularized bone grafts [1,5-7]. Bone grafts with an independent vascular supply demonstrate reliable osteoinductive, osteoconductive, and osteoprogenitor activities [4]. The contralateral untraumatized fibula is an ideal donor site for a vascularized bone graft; however, in cases where microsurgery is not feasible, the ipsilateral fibula is an alternative [2]. In our case, it was not possible to obtain a graft from the contralateral fibula due to the severe crush injury. A previous study reported that united fibular segments were used to reconstruct the defect in cases of associated fibular fractures [8]. In our case, the fractured segments united while we waited for the patient's gen-
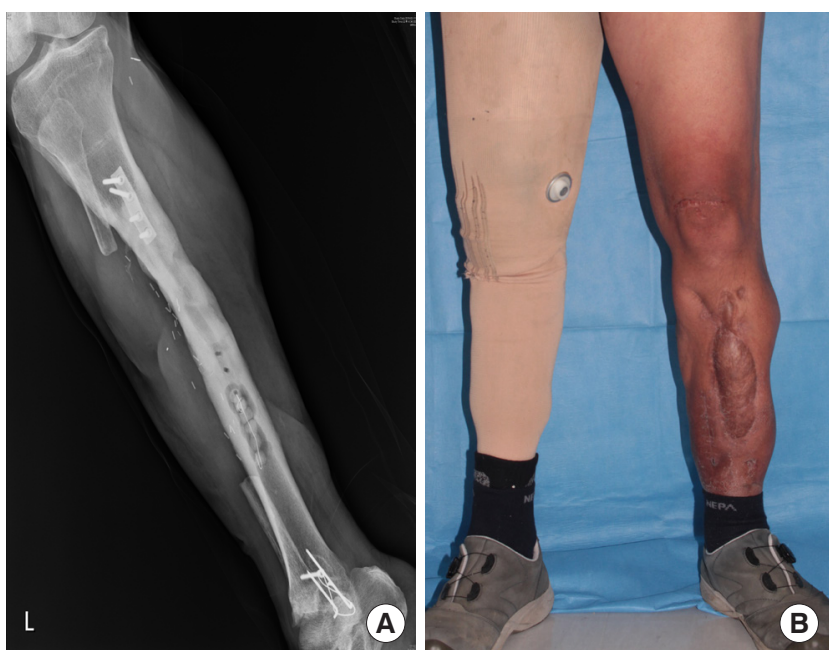

Fig. 4. Twelve years postoperatively, good bony union and fibular hypertrophy were maintained. (A) X-ray photography. (B) Anteroposterior photograph of the left lower limb showing weight-bearing ability.

eral condition to improve and for eradication of the $P$. aeruginosa infection. Therefore, we decided to use the ipsilateral fractured segments. However, we were uncertain of the ability of the fractured segments to bear weight without the contralateral leg. Some studies have reported that an ipsilateral fibular graft may result in the loss of supplementary mechanical support of the tibial graft, thereby increasing limb instability $[4,9]$. We managed the issue of instability by using a brace and prosthesis on the contralateral limb along with a cautious rehabilitation schedule.

Dissection of the fibula while preserving the vasculature is more challenging in a traumatized lower leg than in untraumatized tissue. Moreover, the procedure becomes more difficult in cases of fibular fracture. A study reported that dissection was easier in united fractures than in non-united fractures [8]. In our case, there was sufficient time for union of the fractured fibula.

Previous studies reported that the average time for bony union in a vascularized fibular graft was 3 to 6 months $[4,8]$. In the present case, callus formation was observed 2 months after surgery and fibular hypertrophy was observed after 3 months. Considering the overwhelming stress on the unilateral reconstructed tibia, we gradually initiated weight-bearing exercises for the patient. The patient required sufficient time to familiarize himself with walking using the prosthesis and the reconstructed limb.

A review of the literature on vascularized fibular grafts revealed that the stress fracture rate following complete union was $20 \%$ to $60 \%[4,5]$. In our case, a pure stress fracture occurred 2 weeks after initiation of weight-bearing training. The height and body weight of our patient were $178 \mathrm{~cm}$ and $89 \mathrm{~kg}$, respectively. Bone alignment was maintained through internal fixation. External fixation could be problematic for infection and rehabilitation 
treatment, and intramedullary fixation was not used because it could cause problems with blood circulation and stability of the fibular flap. Nevertheless, a stress fracture occurred, which was thought to be because the prosthetic leg and surgical site were not sufficient to support the weight of $89 \mathrm{~kg}$ due to the contralateral amputation. Therefore, when the same operation is performed in the future, it is considered to be preferable to delay the start of rehabilitation, unlike the protocol for fibular flaps in general. Although we started rehabilitation gradually, the stress from the patient's body weight caused the fracture. The fracture was managed with a long-leg cast for 6 weeks.

By reporting this case, we have highlighted an unusual scenario of unilateral limb weight-bearing, wherein we were able to salvage the sole lower extremity by the ipsilateral fractured fibula transfer and a bipedicled skin flap.

\section{NOTES}

\section{Conflict of interest}

No potential conflict of interest relevant to this article was reported.

\section{Ethical approval}

The study was approved by the Institutional Review Board of Wonju Severance Christian Hospital (IRB No. CR321315) and performed in accordance with the principles of the Declaration of Helsinki. Written informed consent was obtained.

\section{Patient consent}

The patient provided written informed consent for the publication and the use of his images.

\section{Author contribution}

Conceptualization: J Kim. Data curation: SW Kim, ES Kim. Visualization: SW Kim, ES Kim, CE Yang, J Kim. Writing - original draft: ES Kim. Writing - review \& editing: SW Kim, ES Kim. All authors read and approved the final manuscript.

\section{ORCID}

Eon Su Kim

https://orcid.org/0000-0001-5938-9645

Chae Eun Yang

Jiye Kim

https://orcid.org/0000-0001-8128-791X

https://orcid.org/0000-0002-1724-4250

Sug Won Kim

\section{REFERENCES}

1. Taqi M, Raju S. Fibula free flaps. In: StatPearls [Internet]. Treasure Island: StatPearls Publishing; 2021.

2. Chung DW, Han CS, Lee JH. Reconstruction of composite tibial defect with free flaps and ipsilateral vascularized fibular transposition. Microsurgery 2011;31:340-6.

3. Jeng SF, Kuo YR, Wei FC, et al. Concomitant ipsilateral pedicled fibular transfer and free muscle flap for compound tibial defect reconstruction. Ann Plast Surg 2001;47:47-52.

4. May JW Jr, Jupiter JB, Weiland AJ, et al. Clinical classification of post-traumatic tibial osteomyelitis. J Bone Joint Surg Am 1989;71:1422-8.

5. Kovoor CC, Jayakumar R, George V, et al. Vascularized fibular graft in infected tibial bone loss. Indian J Orthop 2011; 45:330-5.

6. Lin $\mathrm{CH}$, Wei FC, Chen HC, et al. Outcome comparison in traumatic lower- extremity reconstruction by using various composite vascularized bone transplantation. Plast Reconstr Surg 1999;104:984-92.

7. Yazar S, Lin CH, Wei FC. One-stage reconstruction of composite bone and soft-tissue defects in traumatic lower extremities. Plast Reconstr Surg 2004;114:1457-66.

8. Chacha PB, Ahmed M, Daruwalla JS. Vascular pedicle graft of the ipsilateral fibula for non-union of the tibia with a large defect: an experimental and clinical study. J Bone Joint Surg Br 1981;63-B:244-53.

9. Swartz WM, Mears DC. Management of difficult lower extremity fractures and nonunions. Clin Plast Surg 1986;13: 633-44. 